\title{
Analysing and Designing Of Traffic Data System Using Big Data
}

\author{
Ranu Munnasingh Thakur ${ }^{1}$, Pranay. D. Saraf ${ }^{2}$, Ravikiran Bhat ${ }^{3}$ \\ ${ }^{l}$ (Department of Computer Science and Engineering, G.H.Raisoni College of Engineering, Nagpur, India) \\ ${ }^{2}$ (Department of Computer Science and Engineering, G.H.Raisoni College of Engineering, Nagpur, India) \\ ${ }^{2}$ (Knowx Innovation Pvt. Ltd., Bangalore, India)
}

\begin{abstract}
Data at segmentation has been helpful tremendously and decidedly to overcome the heterogeneity of the data which is relating to an accident. K-modes clustering modus operandi is considered with respect to entire set of data. Comparison basically between the two of the sets of data fundamentally for the analomalies of the content and the context is herein recognized. Contextual kind anamoly in detection can be worked for the schema. The respective schema is herein evaluated against those of dodgers, dataset which are available in learning kind repository and those of $R$ statistical kind toolbox.
\end{abstract}

Keywords: Big Data, Wireless Sensor Network, Big data, Hadoop, Anamalies

\section{Introduction}

Cluster type analysis does herein reinforce or aid the respective segmentation of the road type accidents. Karlaftis and Tarko herein utilised the cluster type analysis primarily to scrutinise, make to categorisation of the related data and conclude at cluster kind examined results[1]. Utilising Negative kind binomial aids in recognizing the age of driver while at the incident. Ma and Kockelman herein utilized clustering as for the segmentation of the respective data[2]. Poisson type models is better in standard type regression basically when it comes to handling the non-negative, those of random and also discrete kind of features in crash kind counts.[3][4]

Various regression models helps in expediently locating by engineers in traffic department to know the possible happening of an accident at the spot, but it is limited with patterns which may be defacto unanticipated. Conventional kind databases wherein developed utilizing the statistical kind scrutinisation. There could be chance of invalidity when a huge set of data is analysed. Statistical kind of test basically due to sparse type data can be worked herein. As aforementioned models have their own kind assumptions and any breach may end in an error.

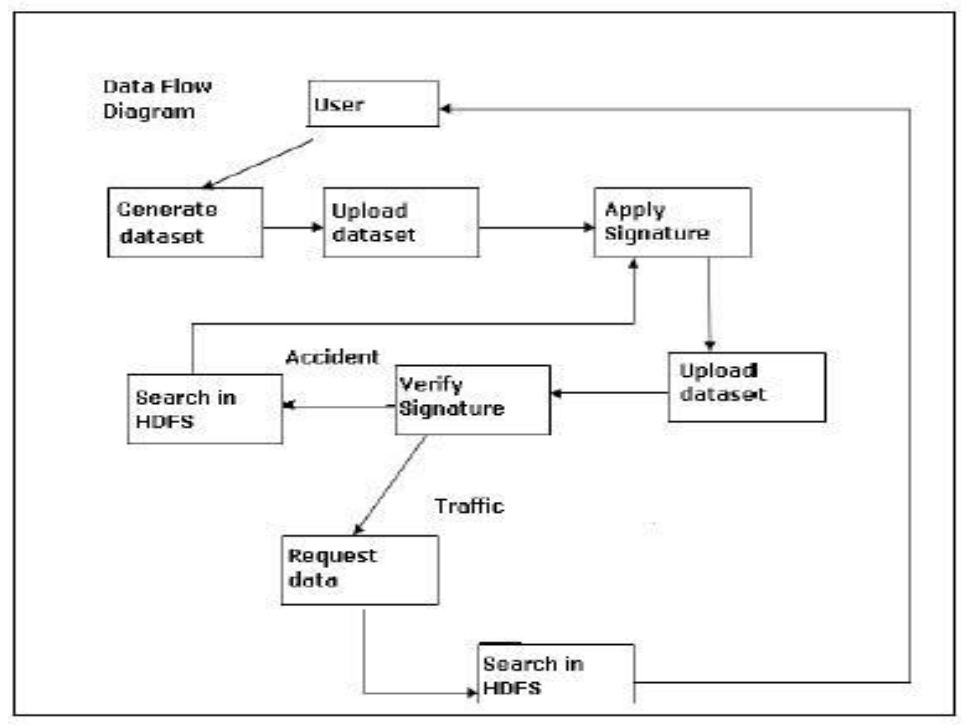

Fig 1: User participation for search

\section{Analysing and Designing Of Traffic Data System Using Big Data}

Chen and Chang [5], herein scrutinsed relevant data utilizing CART and the negative binomial kind regression type model. Abellan et al. [6] examined two lane rural highways. Method of tree was utilized at Spain. Depaire et al. [7] considered latent kind clustering basically on two roads. After gaining with the cluster numbers herein, we utilized the $\mathrm{k}$ type modes of algorithm basically of $\mathrm{R}$, statistical software to bring segmentation for the accidental type data in set. Each of the clusters later is considered for a characterization. 


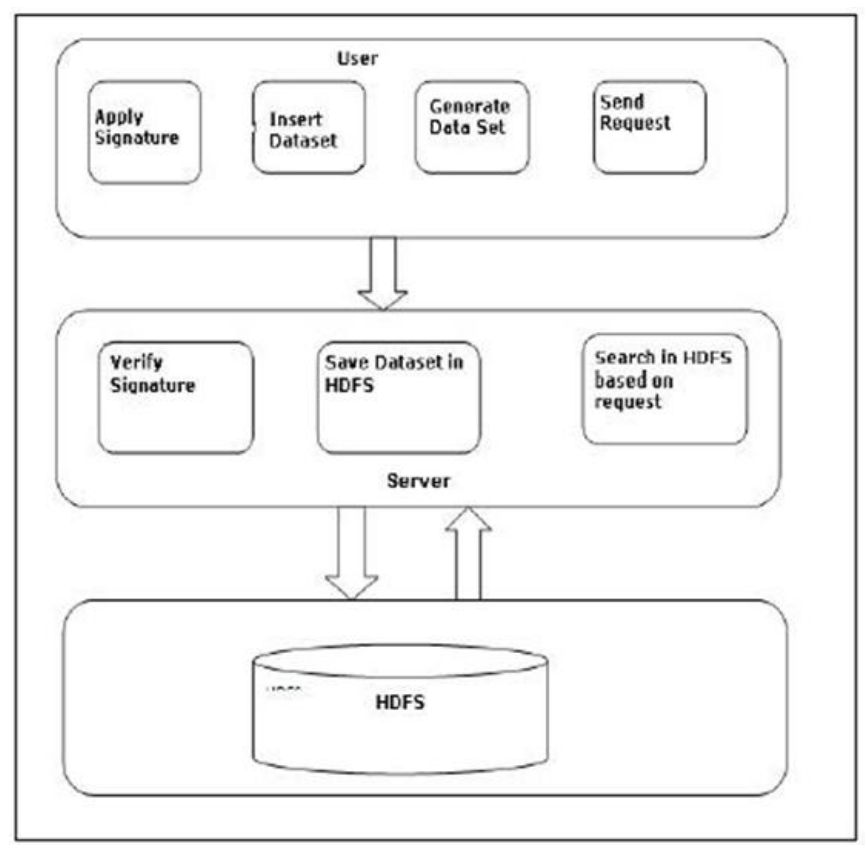

Fig 2: Architectural representation

CART type algorithm was considered in analyzing the respective information basically on records on crash. Cluster kind analysis herein utilized with $\mathrm{K}$ type modes as an algorithm. Processing of respective data deals with expelling out noise, handle those of missing values and taking out attributes which are not relevant. Technique of hierarchical type clustering, $\mathrm{K}$ type modes and LCC kind has been utilized as part of analysis. LCC is widely accepted though. In model kind simulations has equi- kind efficiency observed in both K kind modes and LCC type. [8]. Results from clustering, modes of K type are speedier and efficient kind. Mining of data, wherein association type rule can be recognized as popular kind.[9] especially when it satisfies with the threshold kind criteria. Huge data set herein arrives in together principally to a lesser error as to a prediction, when worked with algorithms, which has training error of accuracy. Motivation of this particular paper is basically to consider embodiment with these expensive type algorithms in order to arrive at the finest data in relevance to accident prone areas. There is restraint for detection in offline. Map reduce can be utilized or divide and conquer can be considered along. [10].

\section{Analysing and Designing Of Traffic Data System Using Big Data}

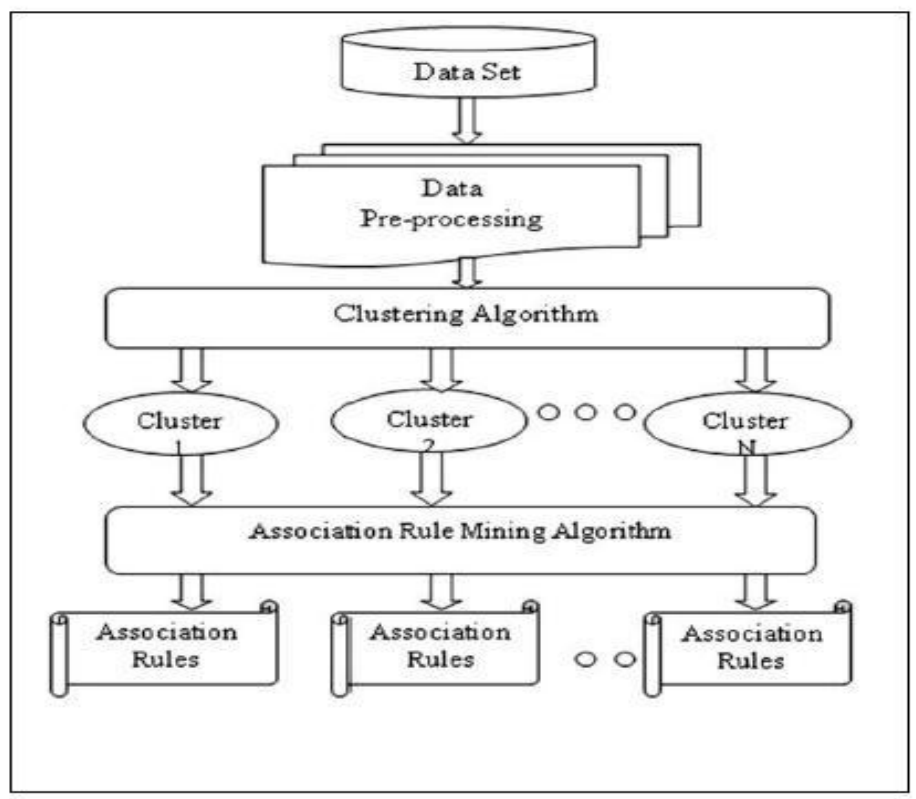

Fig 3: Proposed kind of for considering to analysis. 


\section{Anamalies}

Anamalies of collective data type are viewed basically with other data kind points against those of other type datasets. Contextual kind makes out to find the basic relationship with the respective datasets where there maybe external kind behavior, which can be observed. Discrimination can be arrived at, from the content type anamolies. This is factual that, sensors of Big Data utilize case, where it becomes much difficult to bring out records of entire set or anamolies kind. Lee et al herein has proposed the work principally to detect those of anamolies basically by leveraging those of Hadoop.

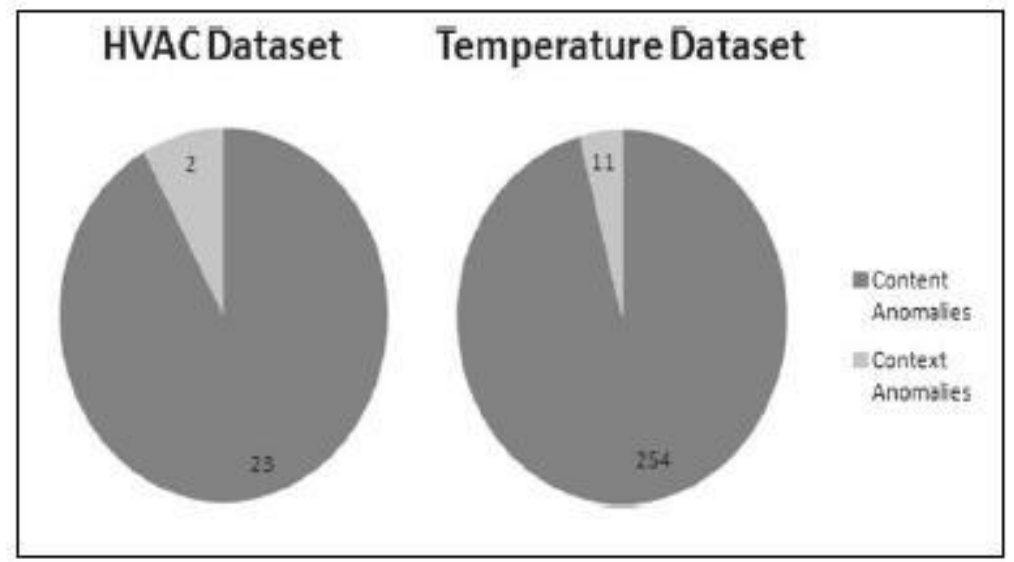

Fig 4: Detection of context

Comparison is made between two of the datasets basically for two of anamolies type which is recognized herein.

\section{Detection Of Anamoly}

Mahapatra et al [13] herein proposed a contextual kind anomoly basically in framework for detection. ALEround et al [14] considers applying contextual type anamoly primarily to bring about zero attacks especially

\section{Analysing And Designing Of Traffic Data System Using Big Data}

At the cyber area. Detection of anamoly is worked with measure of distance. Values relation to anamoly is arrived at. Distance herein is easily not measurable. There is disadvantage with Miller et al[15] , that herein an algorithm, which is defacto a difficult type in consideration. Detection of anamoly has been herein proposed by Xie et el[18] basically with histogram kind approach with networks of wireless and are hierarchical in nature. Multivariate kind of data is herein considered. SVM and the respective neural kind networks requires much time primarily for training and of little testing kind.

\section{Sensors}

Data from sensors, which is streamed from the respective sources, constitutes those of electrical type outlets, pipes of water flow and various others, which defacto follows the corresponding template and huge amount of respective data, which is an input kind in a more frequent manner, can be actually worked. There may be difficulty in coping basically with the velocity and data in respect to volume. Modern developments in technology are focusing at creating new age buildings developed with intelligence. Sensors herein may introduce new kind information which actually diminishes or even enhances the abnormality of the respective anamoly. Calculating the contextual kind anamoly is expensive in terms of computation.

Comparison need to be performed with an incoming value of sensor type with those corresponding kind profiles which comprises the value of incoming with basically an average of respective all sensors in values composing those of sensor type profile, which are corresponding. There are two level of abstraction which can be known herein. Similar kind sensors are amalgamated into those of profiles of sensors and also respective level of context is actually created. Correlation kind of matrix as a method can be considered. Clustering is considered forth in both approaches. 


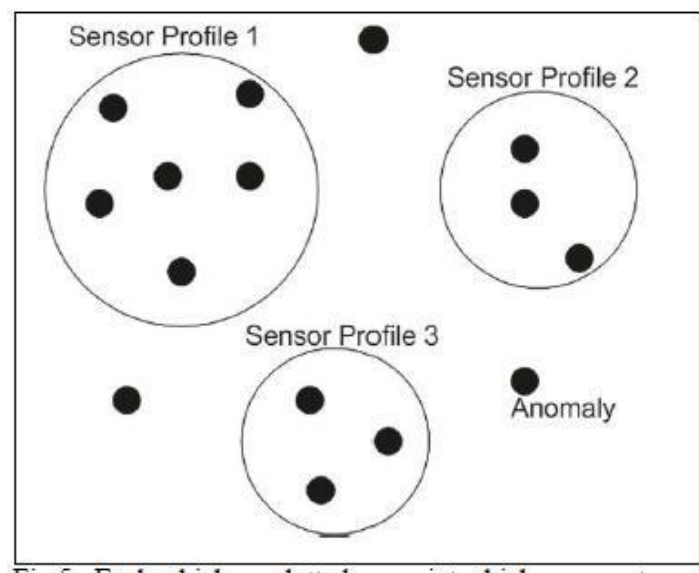

Fig 5: Each which are dotted are point which represents a reading of sensor, circles denote groups of respective sensor or profiles of sensor.

\section{Classification}

Classification based algorithms herein requires labels of accurate type for those normal type classes with those data relating to training. For a multiclass type classification and also anomoly in detection especially in wireless kind sensor networks, Shilton et al [16] actually proposed SVM kind approach. Data type points which herein cannot be classified are recognized as anamolous nature.

\section{Algorithm Worked}

Input: Traffic info from Map reduces Output: time which has less traffic Step1:Filter traffic between 6 to 10 Steps 2:-

Find highest number of vehicle for all traffic info

\{

Analysing And Designing Of Traffic Data System Using Big Data

Find number of vehicle/highest no of vehicle

\}

Step3:

Sort average Step4:

Display time when less no of traffic. If $(\mathrm{val}<0.5)$

\{

Display as good

\}

Else if $(\mathrm{val}<0.8)$

\{

Display as average

\}

Else

\{

Display as bad

\} 
Step5:-Stop

Big Data and other work by Kittler et al[11] herein presents architecture of system basically to detect those anamalies in the respective machine and the domain in perception. Predictor of Bayesian kind is herein improved by notions including outlier, distribution drift, noise and detection of novelty and of rare type events. Detectors which are content and context type are considered herein. Separation does occur basically with regard to vast quantity of data. Central type reposity considers an algorithm with time of testing being too fast. Detector of content can be recognized as false type and positive and even ensures that respective sensor is not defacto producing, herein the results, which are wholly the anamalous type. Training of profiles of sensor can be outlined herein with contextual type. The evaluation of the respective schema is performed utilizing the three data sets. One basically for set of HVAC kind electricity sensors, other basically for the temperature set and the third being related to traffic.

VII. Results

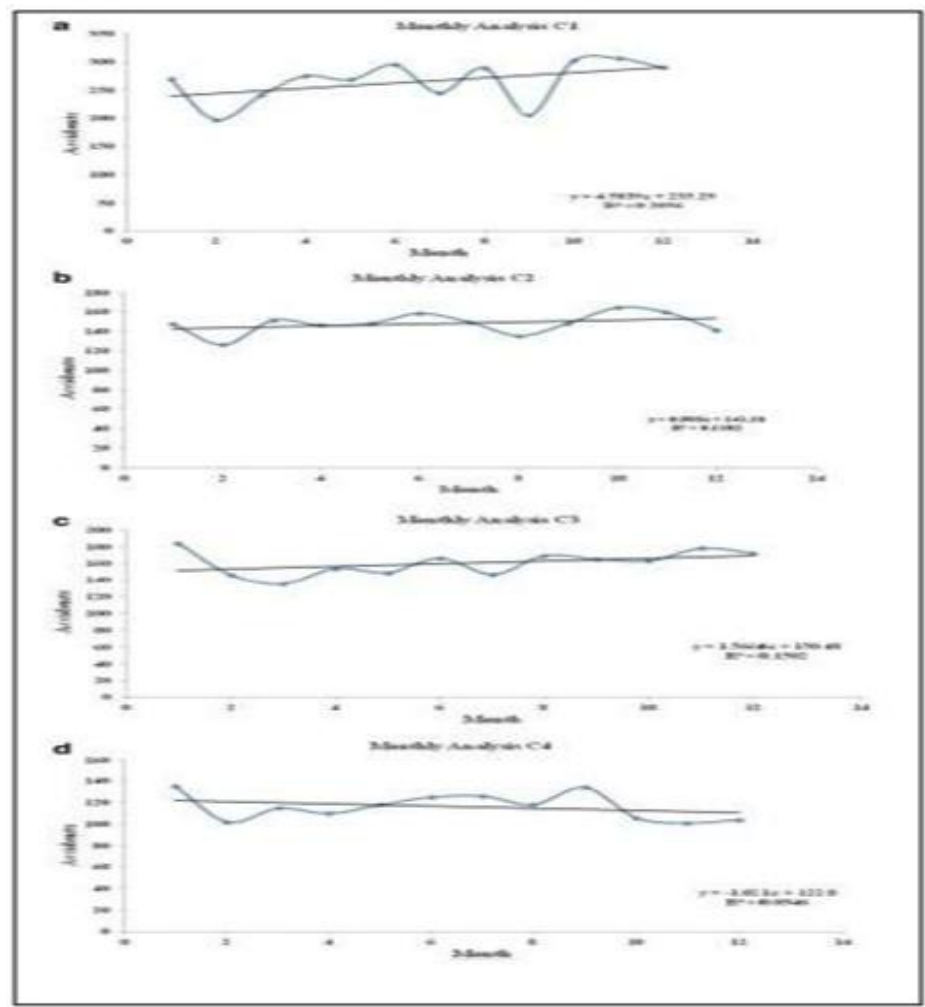

Fig 6: Trend type analysis of respective cluster

\section{Analysing and Designing Of Traffic Data System Using Big Data}

We also consider the trend analysis of all respective clusters and EDS primarily on monthly basis. There are percentages of accidents especially at wheeler. It is those at an intersection, down falling, multiple type vehicles in accidents, hit cases of pedestrian and roll over of vehicles in monthly data calculation, from the respective statistical experts can be known.

\section{Conclusion}

Respective schema is herein favorable enough in detecting positively at real time, those considering content based anamalies. Large set of those anamalous related readings are determined. With Outlier R type, package of statistical nature is competitively performed by the schema. Real time performance can be known with the R kind application. EDS herein does actually reveal precise information.

\section{References}

[1] Karlaftis M, Tarko A. Heterogeneity considerations in accident modeling. Accid Anal Prev.1998;30(4):425-33.

[2] 4. Ma J, Kockelman K. Crash frequency and severity modeling using clustered data from Washington state. In: IEEE Intelligent Transportation Systems Conference. Toronto Canadá; 2006.

[3] Joshua SC, Garber NJ. Estimating truck accident rate and involvements using linear and poisson regression models. Transp Plan Technol. 1990; 15 
[4] Maher MJ, Summersgill I. A comprehensive methodology for the fitting of predictive accident models. Accid Anal Prev Elsevier. $1996 ; 28$.

[5] Chang LY, Chen WC. Data mining of tree based models to analyze freeway accident frequency. J Saf Res Elsevier. 2005;36.

[6] Abellan J, Lopez G, Ona J. Analyis of traffic accident severity using decision rules via decision trees, vol. vol. 40. Expert System with Applications: Elsevier; 2013.

[7] Depaire B, Wets G and Vanhoof K. Traffic accident segmentation by means of latent class clustering, accident analysis and prevention, vol. 40. Elsevier; 2008.

[8] Goodman LA. Exploratory latent structure analysis using both identifiable and unidentifiable models. Biometrica. 1974;62

[9] Agrawal R, Srikant R. Fast algorithms for mining association rules in large databases. In: Proceedings of the 20th International Conference on very large data bases; 1994. pp. 487-99.

[10] Dean J, Ghemawat S (2008) MapReduce: Simplified data processing on large clusters. Commun ACM 51(1):107-113

[11] Kittler J, Christmas W, Campos TD, Windridge D, Yan F, Illingworth J, Osman M (2013) Domain anomaly detection in machine perception: a system architecture and taxonomy. IEEE Trans Pattern Anal Mach Intell 99(PrePrints):1 